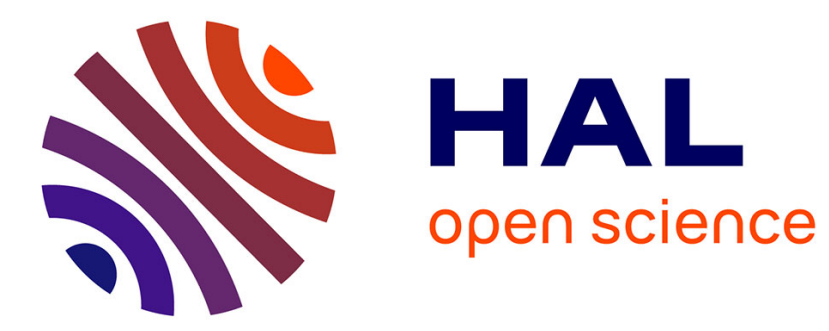

\title{
Snoek-Köster Relaxation. New Insights - New Paradigms
}

\author{
L. Magalas
}

\section{To cite this version:}

L. Magalas. Snoek-Köster Relaxation. New Insights - New Paradigms. Journal de Physique IV Proceedings, 1996, 06 (C8), pp.C8-163-C8-172. 10.1051/jp4:1996834 . jpa-00254642

\section{HAL Id: jpa-00254642 https://hal.science/jpa-00254642}

Submitted on 1 Jan 1996

HAL is a multi-disciplinary open access archive for the deposit and dissemination of scientific research documents, whether they are published or not. The documents may come from teaching and research institutions in France or abroad, or from public or private research centers.
L'archive ouverte pluridisciplinaire HAL, est destinée au dépôt et à la diffusion de documents scientifiques de niveau recherche, publiés ou non, émanant des établissements d'enseignement et de recherche français ou étrangers, des laboratoires publics ou privés. 
JOURNAL DE PHYSIQUE IV

Colloque C8, supplément au Journal de Physique III, Volume 6, décembre 1996

\title{
Snoek-Köster Relaxation. New Insights - New Paradigms
}

\author{
L.B. Magalas \\ University of Mining and Metallurgy, Akademia Górniczo-Hutnicza, AGH, Faculty of Metallurgy and \\ Materials Science, Al. Mickiewicza 30, 30059 Kraków, Poland
}

\begin{abstract}
Common understanding of the Snoek-Köster (SK) relaxation in bcc metals is based on Schoeck's and Seeger's models. The former considers dragging of the cloud of foreign interstitial atoms (FIAs) by a dislocation in terms of the string model. The latter is based on the concept of kink pair formation on dislocations. A new insight into the SK relaxation, however, can be obtained if the coupling model, developed by K. L. Ngai, is used to interpret mechanical loss spectra induced by the motion of dislocations dragging along the Cottrell cloud of FIAs. This phenomenological model opens a new line of thinking in mechanical spectroscopy of metals to describe complex cooperative phenomena. It provides a very general scenario for two distinct regions of the relaxation process: exponential and stretched exponential. This model implies that the relaxation function may continuously cross over from a Debye function to a stretched exponential function. The one-sided Fourier transform of the derivative of the normalized relaxation function gives the same asymmetry of a loss peak as that observed for the carbon SK peak in deformed ultra-high purity $\alpha$-Iron.

This paper is a résumé of the experimental observations of SK relaxation such as the effect of the amount of plastic deformation, of deformation temperature, of conditions for thermal stability of the SK peak, and its broadening. It is concluded that the concept of the cooperative migration of FIAs in the Cottrell cloud elucidates the primary and secondary features of SK peak. Very low value of the activation enthalpy of the stable SK peak detected in deformed ultra-high purity $\alpha$-Iron doped with 25 at. ppm of carbon (i.e. $\mathrm{H}^{\mathrm{SK}}=0.95 \mathrm{eV}$ ) is given. This small value is interpreted in terms of the coupling model. It is concluded that the basic features of the S-K peaks in $\mathrm{Fe}-\mathrm{C}, \mathrm{Nb}-\mathrm{O}$ and $\mathrm{Ta}-\mathrm{O}$ are essentially the same. To test, however, the coupling model in other bcc alloys, special care must be taken to 'design' favourable experimental conditions to reveal secondary features of the SK peak.
\end{abstract}

\section{INTRODUCTION}

This paper is devoted to the Snoek-Köster (SK) relaxation in deformed ultra-high purity $\alpha$-Iron. The following will be presented: three models of SK relaxation (Schoeck [1-3], Seeger [4-7] and Magalas-Ngai $[8,9])$ and selected own experimental data concerning relaxation phenomena related to the interaction of dislocations with mobile foreign interstitial atoms, that is, SK, Dislocation-Enhanced Snoek Effect (DESE) and $M$ peak. The Schoeck model is based on the assumption that dislocation movement can be described according to the string model, the Seeger model is brought about by the kink pair formation (KPF) process on screw dislocations in the Cottrell cloud, whereas the Magalas-Ngai model develops the string model by taking into account interactions between foreign interstitial atoms (FIAs). Two other relaxation phenomena DESE [10-13] and $M$ peak are related to the interaction of dislocations with the FIAs possessing shortrange mobility. Both peaks are observed after plastic deformation simultaneously with the unstable SK peak. The DESE occurs at the same temperature as the Snoek peak $[10,11]$, whereas the $M$ peak occurs around $350 \mathrm{~K}$. There have been no reports on $\mathrm{M}$ peak before in the literature. SK relaxation which is located well above the Snoek peak in bcc metals is generated by the movement of dislocation segments dragging along with them the Cottrell cloud of FIAs. The exact mechanism of SK relaxation has remained for a long time a controversial issue $[2,6,14-16]$. Recently, however, a new insight into the problem has been provided by the application of the coupling model [8, 17-19]. The Magalas-Ngai model [8, 9] was verified with use of experimental data obtained in this laboratory for ultra-high purity $\alpha$-Iron doped with different amount of carbon. Figure 1 shows the location of all the above relaxation peaks, that is, the SK peak (with its components $\mathrm{SK}_{1}, \mathrm{SK}_{2}$ ), $\mathrm{DESE}$, and $\mathrm{M}$ peak. The carbon Snoek peak is also indicated for 


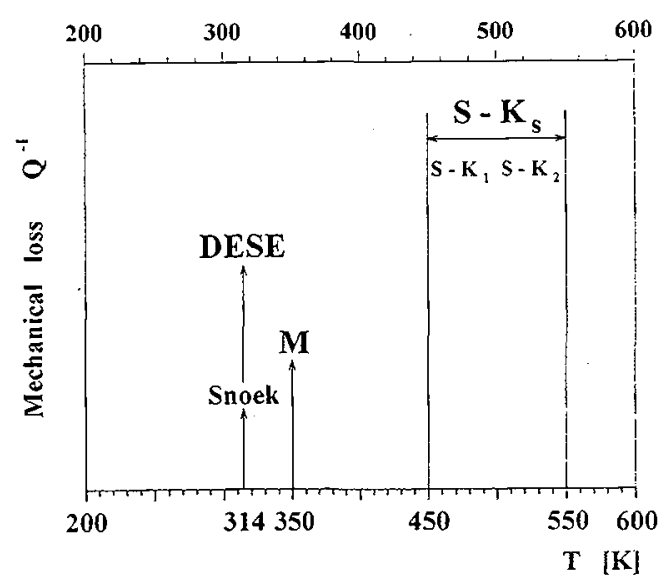

comparison. The Snoek relaxation is the stress-induced short-range statistical redistribution of FIAs residing in octahedral positions in the bcc lattice and creating 'elastic dipoles' around them with a tetragonal strain field [20]. Under the action of an external time-dependent mechanical field the orientation polarization of the elastic dipoles is timedependent, which leads to dissipation of mechanical energy. The activation enthalpy of the Snoek relaxation, $\mathrm{H}^{\mathrm{s}}$, is equal to the migration enthalpy, $\mathrm{H}^{\mathrm{M}}$, of FIAs in the perfect bcc lattice.

Figure 1: Schematic representation of mechanical loss peaks observed in deformed $\alpha$-Iron doped with carbon.

Peak positions correspond to oscillation frequency $f \approx 1.0 \mathrm{~Hz}$.

\subsection{The Schoeck model}

Schoeck's theory of SK relaxation [1-3] is based on the string model for the bowing-out of dislocation segments, dragging the Cottrell atmosphere along with them (see Fig. 2.b). The mechanical loss is given by

The relaxation strength of SK relaxation is given by

$$
\mathrm{Q}^{-1}=\Delta^{\mathrm{SK}} \frac{\omega \tau^{\mathrm{SK}}}{1+\omega^{2} \tau^{2}}
$$

and the relaxation time by

$$
\Delta^{\mathrm{SK}}=\mathrm{Gb}^{2} \mathrm{~L}^{3} / 10 \mathrm{~S}_{\mathrm{d}} \mathrm{V}
$$

$$
\tau^{\mathrm{SK}}=\mathrm{BL}^{2} / 10 \mathrm{~S}_{\mathrm{d}}
$$

where $L$ is the length of the dislocation segments between immobile locking points, $G$ is the shear modulus, $\mathrm{b}$ is the modulus of the Burgers vector, $S_{\mathrm{d}}$ is the dislocation line tension which can be set equal to $\mathrm{Gb}^{2} / \alpha, \mathrm{V}$ is the sample volume, and B is the damping constant of the dislocation motion. The dislocations can only move when they drag their atmosphere along by diffusion of the FIAs. The magnitude of the damping constant is determined by the above process. It is important to emphasize that the damping constant $B$ is expressed with the diffusion coefficient $D_{d}$ of the FIAs in vicinity of the dislocations:

$$
\mathrm{B}=\pi \mathrm{R}^{2} \mathrm{c}_{\mathrm{d}} \mathrm{kT} / \mathrm{b}^{3} \mathrm{D}_{\mathrm{d}},
$$

where $c_{d}$ denotes the concentration of FIAs at the dislocations. Thus, the relaxation time is given by:

$$
\tau^{\mathrm{SK}}=\pi \alpha \mathrm{R}^{2} \mathrm{kTc}_{\mathrm{d}} \mathrm{L}^{2} / 10 \mathrm{~Gb}^{5} \mathrm{D}_{\mathrm{d}} \mathrm{a}_{\mathrm{A}}
$$

where $\alpha$ is a numerical constant of order unity. $D_{d}$ has the Arrhenius temperature dependence $D_{d}=D_{o} \exp \left(-H_{d} / k T\right)$ with $H_{d}$ being the migration enthalpy of the FIAs near the dislocations. The activation enthalpy for migration of the FIAs in close vicinity to a dislocation, $H_{d}$, is higher than $\mathrm{H}^{\mathrm{M}}\left(\mathrm{H}^{\mathrm{M}} \equiv\right.$ $\mathrm{H}^{S}$ ) by an amount which is of the order of the binding energy, $E_{i}: H_{d}=H^{S}+\gamma E_{i}$ [2]. $\gamma=1.5$ for the elastic interaction with screw dislocations, and $\gamma<1$ for non-screw dislocations. Schoeck pointed out that the activation enthalpy of the $\mathrm{SK}$ relaxation $\mathrm{H}^{\mathrm{SK}}$ is a weighted average of values lying within the limits of $\mathrm{H}^{\mathrm{S}}<\mathrm{H}^{\mathrm{SK}}<\mathrm{H}^{\mathrm{S}}+2 \mathrm{E}_{\mathrm{i}}[2]$.

\subsection{The Seeger model}

This model makes strong assumption that only screw dislocations lying along close packed <111> directions with Burgers vector $a_{0} / 2<111>$ are allowed to participate in SK relaxation via KPF process (see Fig. 2.c). Moreover it is also assumed that the mobility of FIAs in the Cottrell cloud controlling the kink mobility is the same as the mobility of FIAs in the perfect, unstressed bcc lattice [4]. The Seeger model predicts the minimum and maximum values of the activation enthalpy of SK relaxation, $H^{S K}$ [6]. Thus the minimum value of $\mathrm{H}^{\mathrm{SK}}$ for the carbon SK relaxation in $\alpha$-Iron can be readily calculated [8]:

$$
\mathrm{H}^{\mathrm{SK}}=\mathrm{H}^{\mathrm{K}}+\mathrm{H}_{\mathrm{K}}{ }^{\mathrm{M}}+\mathrm{H}^{\mathrm{S}}-3 \mathrm{kT}_{\max } / 2=0.43+0.05+0.878-3 \mathrm{k}(461 \mathrm{~K}) / 2=1.298 \mathrm{eV} \text {, }
$$


a
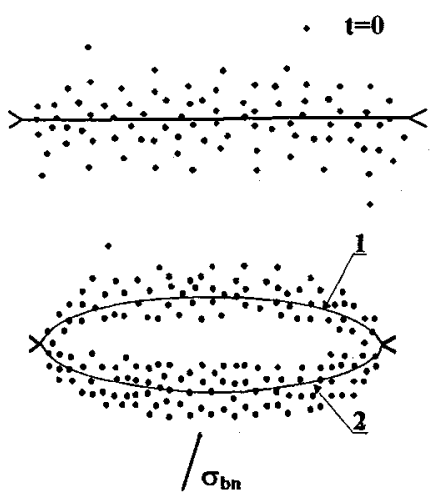

•

$$
b=\frac{a}{2}<111>
$$

c

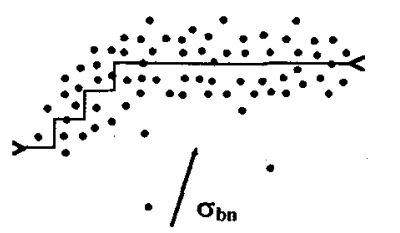

a
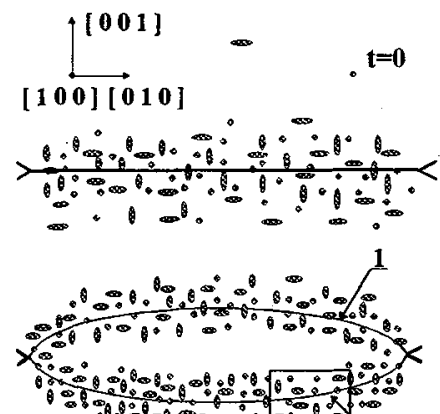

d. 1

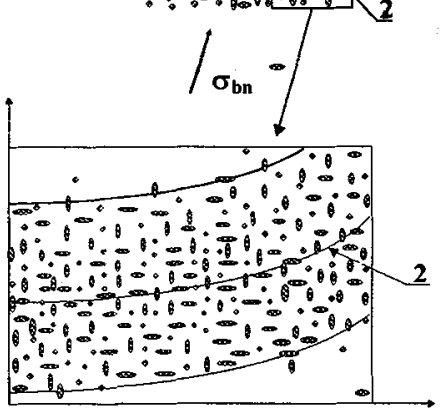

Figure 2: Schematic representation of SK relaxation. (a) The initial state of dislocations at $t=0$, (b) the Schoeck model, (c) the Seeger model, and (d.1, d.2) the Magalas-Ngai model. (d.2) A 'zoom' view on the migration of the FIAs in close vicinity to a dislocation.

where $\mathrm{H}^{\mathrm{K}}$ denotes the enthalpy of formation of kinks on screw dislocations, $\mathrm{H}_{\mathrm{K}}{ }^{\mathrm{M}}$ is the activation enthalpy of kink migration and $\mathrm{T}_{\max }$ is the temperature of the $\mathrm{SK}$ peak. According to Seeger's model, $\mathrm{H}^{\mathrm{SK}}$ cannot be lower than around $1.3 \mathrm{eV}$ in $\mathrm{Fe}-\mathrm{C}$ alloys. This prediction is violated by observations of SK peaks with $\mathrm{H}^{\mathrm{SK}}$ that have values as low as $0.95 \mathrm{eV}$ [8]. Moreover, $\mathrm{H}^{\mathrm{SK}}$ varies over a wide range, decreasing towards the activation enthalpy, $\mathrm{H}^{\mathbf{s}}$, of the carbon Snoek relaxation with a decrease in carbon concentration $[8,9]$. This observation raises serious questions about the validity of the Seeger model based on the KPF mechanism.

\subsection{Coupling model}

This model is based on the concept that dislocations drag Cottrell atmosphere. The model takes into account the cooperative migration of the FIAs caused by two kinds of interactions: the one between the FIAs themselves and the other between the FIAs and time-dependent strain field of dislocations. The above complex phenomenon results in slowing down the rate of migration of the FIAs. For this reason the elastic dipoles were presented in Fig. 2.d with closely located ellipsoids instead of loosely located circles (Fig. 2.b, c). With this model the diffusivity of the FIAs in the Cottrell atmosphere and the activation enthalpy of the SK relaxation can be calculated, its relaxation strength can be accounted for, and the characteristic skew shape of the SK peak can be explained [8].

According to the phenomenological description of the dissipation energy in dynamic loss measurements the complex compliance $S(\omega)$ is given by the one-sided Fourier transform of the derivative of the normalized relaxation function $\psi(t)$, which describes a mechanical relaxation for quasi-static perturbation:

$$
S(\omega)=S_{U}+\int_{0}^{\infty}\left[-\frac{d \psi(t)}{d t}\right] \exp (i \omega t) d t,
$$

where $S_{U}$ is the unrelaxed compliance, and $\omega$ is the angular frequency. For the normalized exponential Debye relaxation function, the complex compliance $S(\omega)$ is readily available analytically $[20,21]$. In general, however, a relaxation function can be considered in the form of the stretched exponential function:

$$
\psi(t)=\exp \left[-(t / \tau)^{\beta}\right]
$$


where $0<\beta \leq 1$. For $\beta \neq 1$ and $\beta \neq 0.5$ the Fourier transform of the derivative of a stretched exponential function must be performed numerically. Figure 3.a shows the normalized relaxation function for different values of $\beta$. The real and imaginary part of the complex compliance $S(\omega)$ is shown in Figs. 3.b and 3.c, respectively. For $\beta \neq 1$ the imaginary part of the complex compliance is asymmetric (see Fig. 3.c). The coupling model describes cooperative relaxation phenomenon in terms of the stretched exponential function $(0<\beta<1)$ and the exponential relaxation function $(\beta=1)$.

a
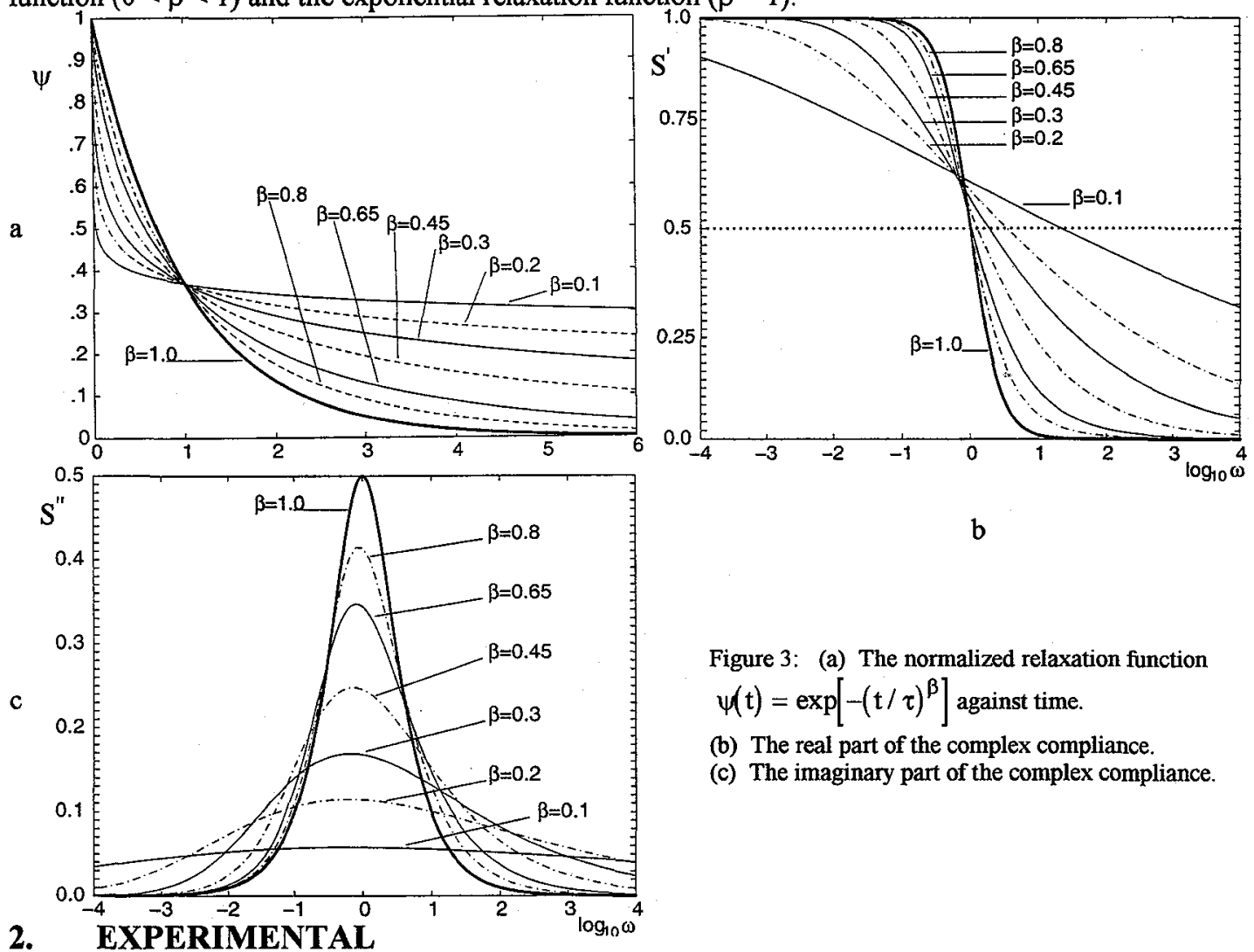

b

Figure 3: (a) The normalized relaxation function $\psi(t)=\exp \left[-(t / \tau)^{\beta}\right]$ against time.

(b) The real part of the complex compliance.

(c) The imaginary part of the complex compliance.

\subsection{Sample preparation}

The samples were prepared from ultra-high purity iron [22]. Iron ingots were pre-doped with 25 and 1000 at. ppm of carbon. The polycrystalline wires were drawn to 1.0 and $0.6 \mathrm{~mm}$ in diameter and $80 \mathrm{~mm}$ in length, then recrystallized for 5 hours at $823 \mathrm{~K}$ in the atmosphere of pure helium and saturated by rapid cooling to $273 \mathrm{~K}$. Carbon pre-doped saturated samples will be referred to as $\mathrm{Fe}-\mathrm{C} / 25 /$ and $\mathrm{Fe}-\mathrm{C} / 1000 /$. The carbon in $\mathrm{Fe}-\mathrm{C} / 1000 /$ was predominantly in the form of large precipitates of cementite. Around 110 at. ppm was dissolved in ferrite as can be inferred from the height of the carbon Snoek peak after saturation. In $\mathrm{Fe}-\mathrm{C} / 25 /$ carbon concentration in solid solution was around $5-10$ at. ppm.

A deformation texture of $\{110\}<111>$, typical for bcc metals, was observed in the investigated samples

\subsection{Mechanical loss measurements}

The loss of mechanical energy and oscillation frequency was calculated either from the Hilbert transformation [23] or from the fast Fourier transform of free decaying oscillations. It is noteworthy that the logarithmic decrement $\delta$ can be calculated with higher precision from the Hilbert transformation than from the classical analysis of decaying amplitudes of oscillations [23]. Fig. 4.b shows the 3-D Nyguist plot of a damped cosine function of Fig. 4.a. The envelope a(t) of decaying oscillations is shown in Fig. 4.c. A plot of $\log [a(t)]$ versus time readily yields the value of the logarithmic decrement (see Fig. 4.d). 

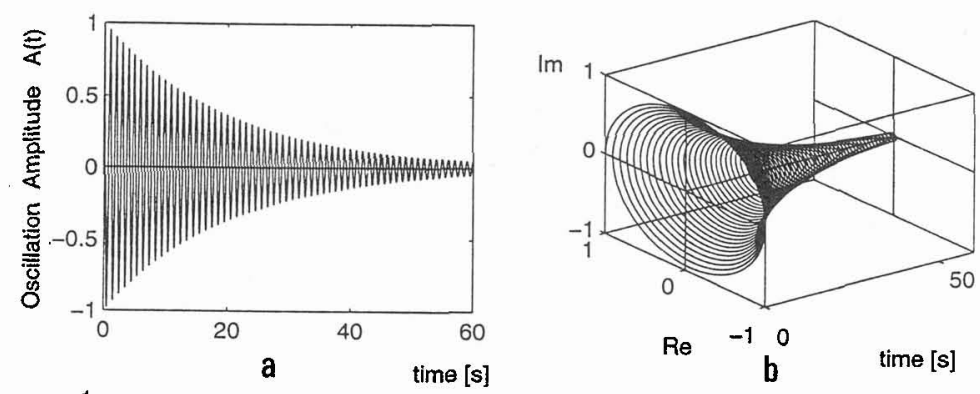

Figure 4: The Hilbert transformation of damped harmonic oscillations $A(t)=\exp \left(-\delta f_{0} t\right) \cos (\omega t)$ for $\delta=5 \cdot 10^{-2}$ and $f_{o}=1.0 \mathrm{~Hz}$.
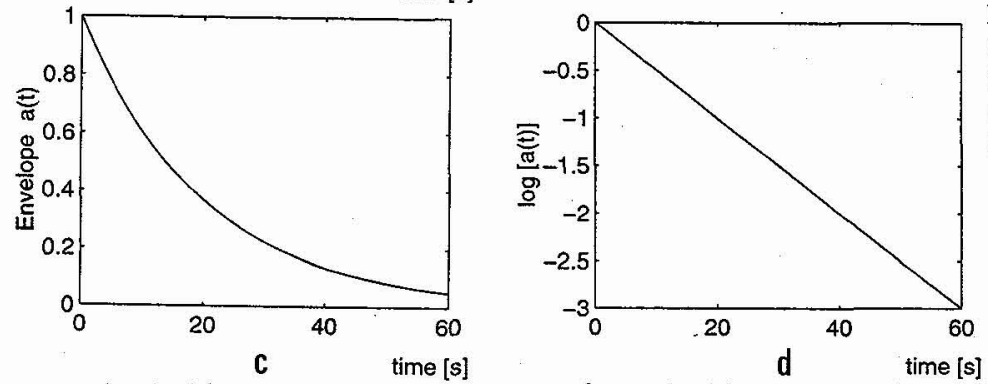

(a) Damped oscillations,

(b) perspective Nyguist plot of the Hilbert transformation of damped oscillations shown in Fig. 4.a, (c) envelope $a(t)$ of damped oscillations, and (d) plot of $\log [a(t)]$ vs time.

The mechanical loss measurements were performed with a constant heating or cooling rate of $200 \mathrm{~K} / \mathrm{h}$ and with a maximum strain amplitude of $8 \times 10^{-6}$. An axial magnetic field of 140 Oe was applied in order to suppress magnetomechanical damping. Cold work (CW) was performed in torsion at room temperature $(\mathrm{RT})$ inside a mechanical spectrometer (inverted torsion pendulum). SK peak, DESE and M peak were detected according to the following procedure. After CW at RT the first mechanical loss measurement of $\mathrm{Q}^{-1}(\mathrm{~T})$ as a function of temperature, referred to as the first up-run, was carried out from $100 \mathrm{~K}$ up to the so-called annealing temperature, $T_{a}$. The second measurement was carried out on cooling down from $T_{a}$ to RT immediately following the first up-run. The investigated samples were also subjected to additional $\mathrm{CW}$ after the above mentioned procedure, that is, after the first up-run followed by the down-run.

\section{RESULTS}

\subsection{The Snoek-Köster relaxation}

The effect of CW at RT on mechanical loss spectra in Fe-C/1000/ is shown in Fig. 5. The shape of the SK peak obtained in the up-run measurements performed just after $\mathrm{CW}$ is different from that obtained in the subsequent down-run and in the next up-run. The SK peak obtained after CW at RT in the first up-run is identified as an unstable SK peak (open symbols). The unstable SK peak changes into the stable SK peak, usually higher, which shifts to lower temperatures in the second down-run (curves 3,4 , 5; filled symbols). A transformation of unstable into stable SK peak in $\alpha$-Iron was first reported by Magalas $e t$ al. [24] and confirmed later by other workers $[25,10,26]$. The carbon Snoek peak disappears after the first up-run indicating that no carbon atom was left in solid solution after annealing, during mechanical loss measurement, up to $\mathrm{T}_{\mathrm{a}}$. The strong zero-point drift (ZPD) is observed in the first up-run and after additional CW over a wide temperature range. It can be concluded that the unstable SK peak is usually accompanied by ZPD (see Figs. 5, 6). ZPD disappears in the down-run while the unstable SK peak transforms into the stable SK peak. The relaxation strength of the stable SK peak and the ZPD increases with the amount of $\mathrm{CW}$ at $\mathrm{RT}$ or $\mathrm{CW}$ at $77 \mathrm{~K}$. It is important to point out that SK peak may evolve during the mechanical loss measurements. Therefore it is necessary to establish optimal experimental conditions for heat treatment, deformation, and mechanical loss measurements in order to observe thermally stable SK peak [9]. An example of perfectly stable SK peak obtained in $\mathrm{Fe}-\mathrm{C} / 1000 /$ after $13 \% \mathrm{CW}$ at $\mathrm{RT}+6 \%$ CW at RT is shown in Fig. 7.

Figure 8 shows the effect of carbon concentration on the stable SK peak after $13 \%$ of CW at RT for $\mathrm{Fe}-\mathrm{C} / 25 /$ (curve 1), and for $\mathrm{Fe}-\mathrm{C} / 1000 /$ (curve 2). For lower concentration of carbon atoms in solid solution the peak temperature, $\mathrm{T}_{\max }^{\mathrm{SK}}$, is shifted towards low temperatures by more than $100 \mathrm{~K}$. 


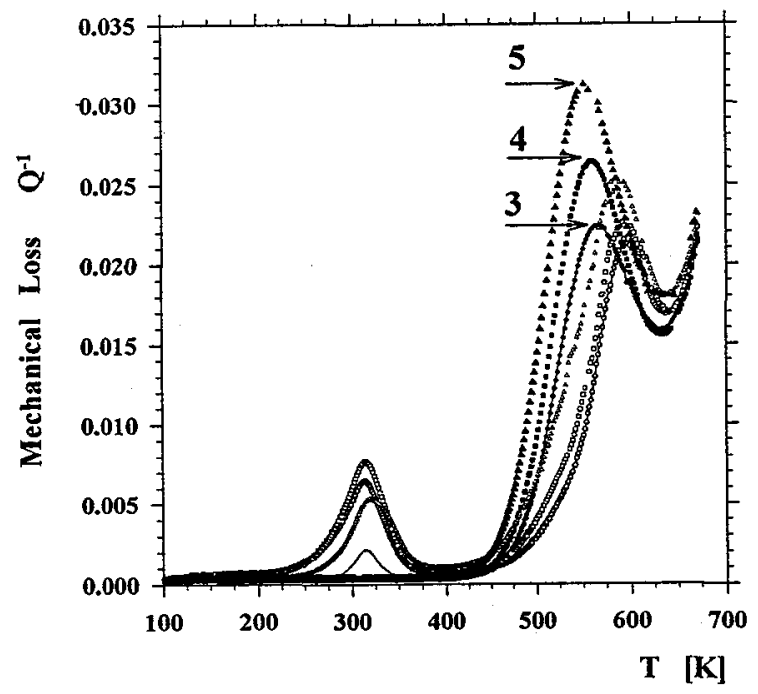

Figure 5: Transformation of unstable SK peak into stable SK peak in Fe-C/1000/ after CW at RT. The curves marked with open symbols were measured with increasing temperature after CW (up-run); unstable SK peak, $T_{a}=673 \mathrm{~K}$. Curves $3,4,5$ were measured with decreasing temperature (filled symbols) - stable SK peak (down-run). A small solid curve represents the carbon Snoek peak around $314 \mathrm{~K}(\mathrm{f} \approx 1 \mathrm{~Hz})$ after saturation from $823 \mathrm{~K}$.

Curve 3 - Stable SK peak after $7 \% \mathrm{CW}$ at RT; unstable SK peak - 0 .

Curve 4 - Stable SK peak after 13\% CW at RT; unstable SK peak - $\square$.

Curve 5 - Stable SK peak after $17 \% \mathrm{CW}$ at RT; unstable SK peak - $\Delta$.
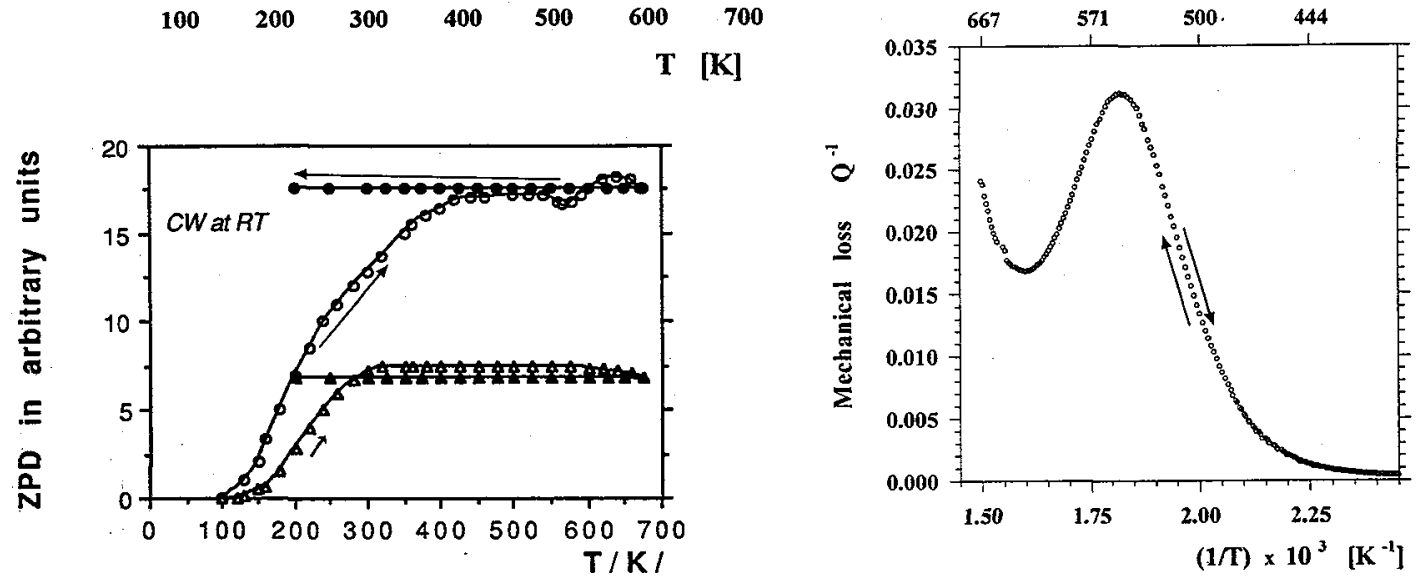

Figure 6: Zero point drift (ZPD) after $7 \% \mathrm{CW}$ at RT and after additional $6 \% \mathrm{CW}$ at $\mathrm{RT}$.

O $7 \% \mathrm{CW}$ at RT up-run, down-run, $\Delta+6 \% \mathrm{CW}$ at RT up-run, $\Delta$ down-run. $\quad \mathrm{T}[\mathrm{K}]$

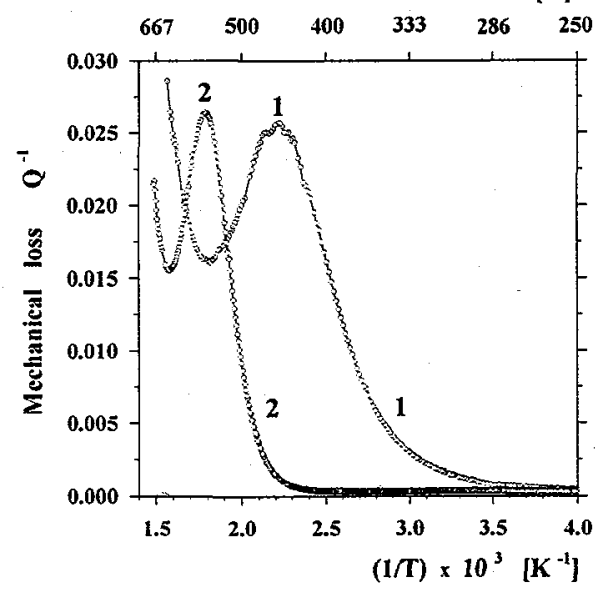

Figure 8: Effect of carbon concentration on SK. peak after $13 \% \mathrm{CW}$ at RT.

Curve 1 - Fe-C/25/, curve 2 - Fe-C/1000/.

Figure 7: Stable SK peak in Fe-C/1000/ after $13 \% \mathrm{CW}$ at RT and additional $6 \% \mathrm{CW}$ at $\mathrm{RT}$.

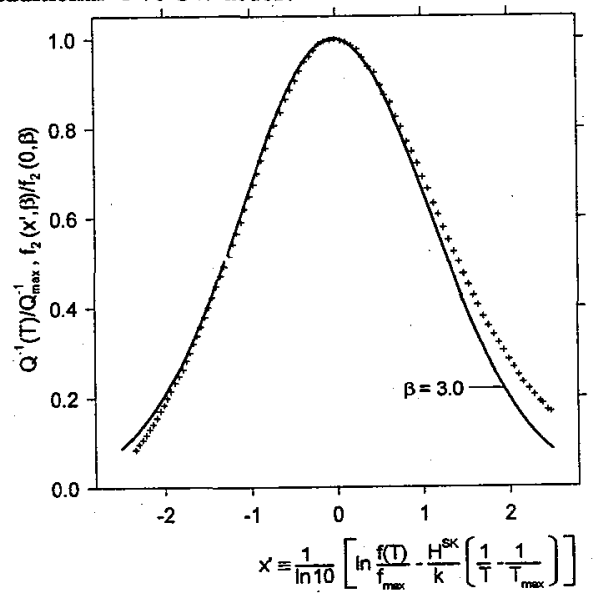

Figure 9: Asymmetrical broadening of stable carbon SK peak in $\mathrm{Fe}-\mathrm{C} / 1000 /$ after $19 \% \mathrm{CW}$ at RT.

$\mathrm{T}_{\max }^{\mathrm{SK}}=550.6 \mathrm{~K}(\mathrm{f} \approx 1.0 \mathrm{~Hz}), \beta=3.0 .(+)$ represents the experimental points, the solid curve represents the theoretical function $[20] \mathrm{f}_{2}\left(\mathrm{x}^{\prime}, \beta\right) / \mathrm{f}_{2}(0, \beta)$ for $\beta=3.0$. 
It should be pointed out that the SK peaks, shown in Figs. 5, 7, 8, are much broader than a Debye peak with a single relaxation time. Broadening of the stable SK peak was calculated on an assumption that symmetrical broadening is caused by a lognormal distribution of relaxation times [20] due to a distribution of dislocation loop lengths (see Fig. 9). $\beta$ denotes here a parameter of lognormal distribution which characterizes the width of the SK peak in comparison with a Debye peak governed by a single relaxation time. Figure 9 also shows a deviation from symmetrical broadening, which occurs on the low temperature (or high frequency) side (see also Fig. 3.c).

\subsection{The DESE and the $M$ peak}

The height of the peak at $314 \mathrm{~K}$ increases with the amount of $\mathrm{CW}$ in $\mathrm{Fe}-\mathrm{C} / 1000$ / (see Fig. 5). This peak is composed of the carbon Snoek peak and the carbon DESE. By contrast, the strong DESE occurs in the first up-run without participation of the Snoek peak in $\mathrm{Fe}-\mathrm{C} / 25 /$ after $13 \% \mathrm{CW}$ at RT (Fig. 10.1, curve a). The initial Snoek peak is represented by curve 0 . Let us note that the height of the DESE would correspond to around 560 at. ppm of carbon atoms in solid solution if this peak were due to the Snoek peak. This is not possible in the investigated samples doped with 25 at. ppm of carbon. This provides a convincing proof of existence of the DESE in $\alpha$-Iron. It can be concluded that the DESE, which is caused by an interaction of short-range mobile FIAs with dislocations, is favoured by small concentration of FIAs. After additional $6 \% \mathrm{CW}$ at RT the M peak occurs around $350 \mathrm{~K}$ together with two constituent SK peaks: $\mathrm{SK}_{1}$ at around $460-470 \mathrm{~K}$ and $\mathrm{SK}_{2}$ around $520 \mathrm{~K}$ (Fig. 10.2, curve b). The M peak also occurs after additional $\mathrm{CW}$ at $77 \mathrm{~K}$ (Fig. 11, curve b). The $\mathrm{M}$ peak anneals out in its temperature range due to pinning by FIAs, as shown in Fig. 11 (increase in $\mathrm{f}^{2}$ ). It is concluded that the $\mathrm{M}$ peak occurs after additional $\mathrm{CW}$, which destroys stable Cottrell atmosphere formed during previous mechanical loss measurement up to $T_{a}$.

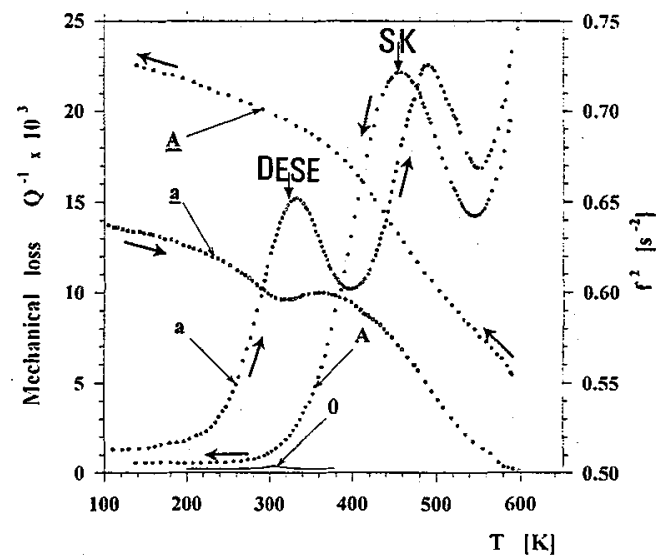

Figure 10.1: DESE and unstable SK peak observed in the up-run after $13 \% \mathrm{CW}$ at RT (curve a). Stable SK peak in the down-run (curve A). Curve 0 represents the carbon Snoek peak.
Figure 11: Effect of $15 \mathrm{~min}$. annealing at $367 \mathrm{~K}$ on $\mathrm{Q}^{-1}$ and modulus $\left(\propto \mathrm{f}^{2}\right)$ on M peak obtained in Fe-C/1000/ after $5 \% \mathrm{CW}$ at $\mathrm{RT}+5 \% \mathrm{CW}$ at $77 \mathrm{~K}$.

The height of the initial carbon Snoek peak observed after saturation is indicated by an arrow.

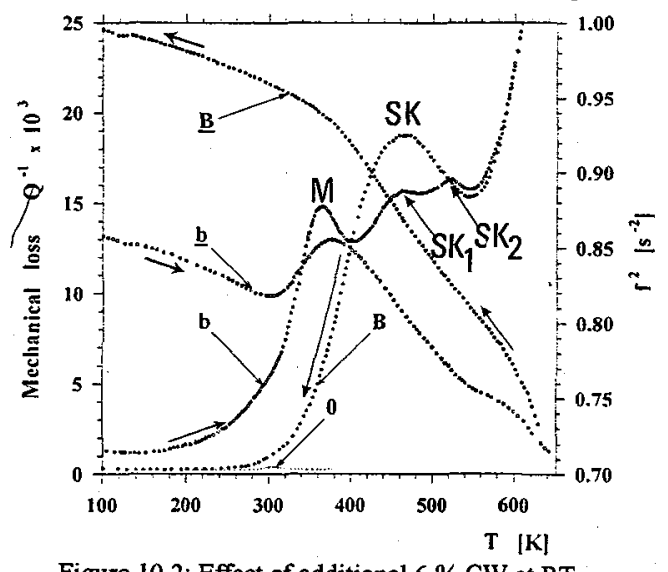

Figure 10.2: Effect of additional $6 \% \mathrm{CW}$ at RT. $\mathrm{DESE}, \mathrm{M}$ peak, and $\mathrm{SK}_{1}, \mathrm{SK}_{2}$ in the up-run (curve b). Stable SK peak in the down-run (curve B).

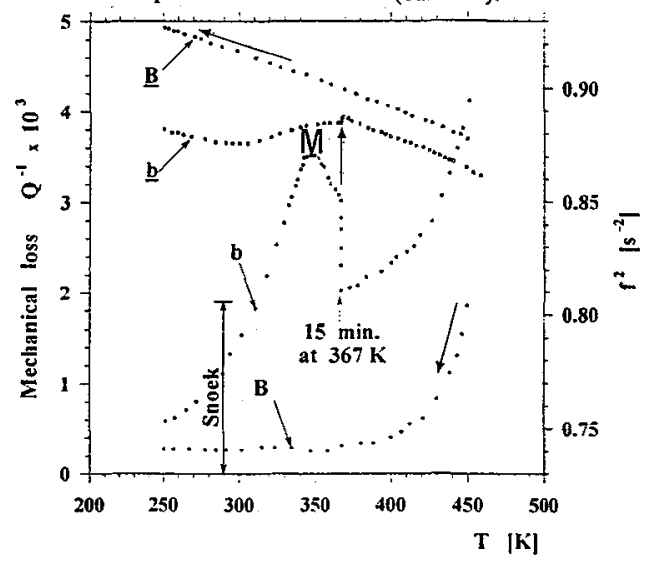




\section{DISCUSSION}

\subsection{Snoek-Köster relaxation}

The succession of unstable and stable SK peaks was found in the whole investigated plastic deformation range. For this reason the appearance of both unstable and stable SK peaks can be considered as a characteristic feature of the SK relaxation in $\alpha$-Iron. To observe a highly stable SK peak one has to work under favourable experimental conditions, which maintain a balance between carbon bound in stable precipitations, trapped at dislocations, and left in solid solution under thermal equilibrium. The transformation of an unstable SK peak into a stable one is explained by assuming that formation of the Cottrell atmospheres occurs during the first mechanical loss measurements in high temperature range. After redeformation of such sample the DESE, $\mathrm{M}$ and two constituent $\mathrm{SK}$ peaks $\left(\mathrm{SK}_{1}, \mathbf{S K}_{2}\right)$ can be observed. Splitting of the SK peak into two constituent peaks can be easily obtained in $\mathrm{Fe}-\mathrm{C} / 25 /$. Similar behaviour was observed in $\mathrm{Nb}-0 / 965 /$ samples, annealed at $\mathrm{T}_{a}=750 \mathrm{~K}$ and then deformed [7]. The relaxation strength of the SK peak, and the M peak increases with the amount of plastic deformation. The relaxation strength of the SK peak increases proportionally to the density of dislocation segments involved in SK relaxation and to the square of the loop length [1]. An increase in plastic deformation induces a strong increase of dislocation density at the expense of the loop length. This is a classical behaviour of the SK peak, which is consistent with the theoretical model of Schoeck [1-3]. Fig. 5 also shows that for constant carbon concentration the peak temperature, $\mathrm{T}_{\max } \mathrm{sK}$, decreases with an increase in plastic deformation. Again, in Schoeck's model this effect results from diminution of the loop length after higher deformations. Since the relaxation time of the SK peak depends on the loop length $\left(\tau^{\mathrm{SK}} \propto \mathrm{L}^{2}\right)$ a decrease in $\mathrm{L}$ results in an evident decrease in the peak temperature, $\mathrm{T}_{\max }{ }^{\mathrm{SK}}$. Moreover, the peak temperature, for the same amount of deformation, depends strongly on the concentration of carbon atoms (Fig. 8). We recall that CW at RT is more effective in generation of the SK peak than low temperature deformation $[10,24]$. The SK peak is much broader than a single Debye peak (Figs. 5, 7-9). Some part of the symmetrical peak broadening is probably due to a distribution of loop lengths. Another source of broadening of the SK peak originates from the cooperative migration dynamics of FIAs in the Cottrell cloud. Therefore the skew asymmetry of the SK peak is observed. The skewness of the SK peak, among other characteristics, provides evidence for the presence of cooperative motion of FIAs in the mechanism of SK relaxation, which together with the distribution of loop lengths accounts for the SK peak broadening (see Fig. 9).

The measured activation enthalpy of the SK relaxation, $\mathrm{H}^{\mathrm{SK}}$, in high-purity iron containing carbon varies from $1.46 \mathrm{eV}$ to $1.91 \mathrm{eV}$ while the preexponential factor $\tau_{\infty}{ }^{\mathrm{SK}}$ varies between $10^{-15}$ and $10^{-19} \mathrm{~s}[8,9,24$, 26]. The relaxation parameters of the SK peak strongly dependent on experimental conditions which give rise to different dislocation structures (dislocation densities) and Cottrell cloud densities, $c_{d}$. Nevertheless, $\mathrm{H}^{\mathrm{SK}}$ is always higher than the activation enthalpy of the carbon Snoek peak in iron $\mathrm{H}^{\mathrm{S}}=0.878 \mathrm{eV}$ [27]. Similar observations were made for other bcc metals [7]. The Arrhenius plot for the SK peak in Fe-C/25/ after $13 \% \mathrm{CW}$ yields the very small value $\mathrm{H}^{\mathrm{SK}}=0.95 \mathrm{eV}$ and the very large value $\tau_{\infty}^{\mathrm{SK}}=10^{-12} \mathrm{~s}[8]$

\subsection{The coupling model}

The Magalas-Ngai model [8] is based on Schoeck's original model [1-3], which presents SK relaxation as caused by dislocation movement controlled by viscous diffusion of FIAs locked in time-dependent dislocation stress field. The coupling model takes into account the fact that the local concentration of FIAs in the Cottrell atmosphere is much higher than the concentration of FIAs in solid solution. Also, the interactions between the FIAs in the Cottrell atmosphere are stronger than the long range interactions between FIAs in the perfect bcc lattice. When the density of interacting species is high, as in the case of the Cottrell atmosphere, the most probable relaxation time, $\tau^{*}=\tau_{\infty}{ }^{*} \exp \left(\mathrm{E}_{\mathrm{a}}{ }^{*} / \mathrm{kT}\right)$, defined as the reciprocal of the peak angular frequency, has an activation enthalpy higher than the energy barrier for non-interacting (isolated) relaxing species. At microscopically short times, an irreversible process described by a relaxation function will follow purely exponential law: $\exp \left[-\left(t / \tau_{0}\right)\right]$. However, there exists a time scale, $t_{c}$, beyond which the initial diffusion rate, $W_{0}$, slows down. Diffusion leads to slowing down of the rate according to $W_{o}\left(t / t_{c}\right)^{-n}$; the relaxation function, after averaging over all the relaxing species, now assumes the stretched exponential form, $\exp \left[-\left(t / \tau^{*}\right)^{1-n}\right]$. The quantity $n$ appearing as the exponent is called the 
coupling parameter, which is a measure of the slowing down caused by the interactions. Thus the normalized relaxation function, $\psi(t)$, assumes two different forms in two separate time regimes:

$$
\psi(t)= \begin{cases}\exp \left[-\left(t / \tau_{0}\right)\right] & \text { for } t<t_{c}, \\ \exp \left[-\left(t / \tau^{*}\right)^{1-n}\right] & \text { for } t>t_{c}\end{cases}
$$

with a cross-over between them occurring at $t=t_{c}$. Continuity, required of the relaxation at all times, when applied at $t=t_{c}$, gives $\exp \left[-\left(t_{c} / \tau_{o}\right)\right]=\exp \left[-\left(t_{c} / \tau^{*}\right)^{1-n}\right]$, providing the so-called 'second' relation:

$$
\tau^{*}=\left[t_{c}^{-n} \tau_{o}\right]^{1 /(1-n)}
$$

$\mathrm{n}$ determines the degree of the departure of the form of the relaxation function from the Debye relaxation and its effective relaxation time $\tau^{*}$ from the Debye relaxation time $\tau_{0}$. The Cottrell atmosphere with concentration $c_{d}$ of FIAs can be considered as a region where the FIAs interact with the dislocations and among themselves. Consequently, the diffusivity of FIAs in the Cottrell cloud must differ from that in the unstressed lattice.

- For $\mathrm{t}<\mathrm{t}_{\mathrm{c}}$, migration and reorientation of elastic dipoles in the Cottrell cloud proceeds independently, without the presence of other FIAs as yet being felt, as if it were like the classical Snoek relaxation of noninteracting elastic dipoles in an unstressed bcc lattice. Interactions between FIAs are of atomic origin and hence $t_{c}$ is expected to be a microscopic time, possibly of the order of a pico second as observed in other materials. Therefore behaviour in the short time regime, even though it actually takes place, will not be seen in experiments performed in the long (macroscopic) time regime.

- For $t>t_{c}$, migration and reorientational relaxation of each elastic dipole is slowed by the interactions between the elastic dipoles (see Fig. 2.d.2). The independent relaxation rate $\mathrm{W}^{\mathrm{S}}$ is slowed. Consequently, the relaxation rate, after being averaged over all the relaxing dipoles, has the time-dependent form $W^{S}(t) \propto W^{S}\left(t / t_{c}\right)^{-n}$, where $n$ is a coupling parameter reflecting the degree of correlation or cooperation in the relaxation process arising from mutual interactions of FIAs in the Cottrell cloud. The relaxation function becomes a fractional exponential, $\psi^{\text {Cottrell }}(t)=\exp \left[-\left(t / \tau_{\text {Cottrell }}^{*}\right)^{1-n}\right]$, with its effective relaxation time related to $\tau^{\mathrm{s}}$ by $\tau_{\text {Cottrell }}^{*}=\left[\mathrm{t}_{\mathrm{c}}{ }^{-\mathrm{n}} \tau_{\infty}^{\mathrm{S}} \exp \left(\mathrm{H}^{\mathrm{S}} / \mathrm{kT}\right)\right]^{1 /(1-\mathrm{n})}$. The effective diffusion coefficient, $\mathrm{D}_{\text {Cottrell }}^{*}$, of the FIAs in the Cottrell cloud can now be readily obtained from $\tau_{\text {Cottrell in the form: }}^{*}$ $\mathrm{D}_{\text {Cottrell }}^{*}=6 \mathrm{a}^{2} / \tau_{\text {Cottrell }}^{*}$, where $\mathrm{a}$ is the characteristic jump distance of the FIAs. All the basic equations of Schoeck's string model remain valid in our model of SK relaxation except that in the expressions for the relaxation times $D_{d}$ is replaced by $D_{\text {Cottrell }}^{*}$ The interactions between the FIAs in the Cottrell cloud have reduced their diffusion coefficient through an increase in the relaxation time $\tau_{\text {Cottrell }}^{*}$.

However the time dependence of the relaxation function for migration of the FIAs has no effect on the frequency dependence of the SK relaxation because here it was assumed that the relaxation strength of the SK peak comes entirely from the movement of dislocation loops.

In this manner a modified version of the Schoeck model was obtained by using the coupling model, which takes into account the interacting Cottrell cloud:

$$
\tau_{\mathrm{av}}^{\mathrm{SK}}=\frac{\pi \alpha \mathrm{R}^{2} \mathrm{kTc}_{\mathrm{d}} \mathrm{L}_{\mathrm{av}}{ }^{2}}{10 \mathrm{~Gb}^{5} \mathrm{D}_{\text {Cottrell }}^{*}}=\frac{\pi \alpha \mathrm{R}^{2} \mathrm{kTc}_{\mathrm{d}} \mathrm{L}_{\mathrm{av}}^{2}\left[\mathrm{t}_{\mathrm{c}}{ }^{-\mathrm{n}} \tau_{\infty}^{\mathrm{S}}\right]^{1 /(1-n)}}{10 \mathrm{Ga}^{2} \mathrm{~b}^{5}} \exp \left[\frac{\mathrm{H}^{\mathrm{S}}}{(1-\mathrm{n}) \mathrm{kT}}\right] \equiv \tau_{\infty, \mathrm{av}}^{\mathrm{SK}} \exp \left[\frac{\mathrm{H}^{\mathrm{S}}}{(1-\mathrm{n}) \mathrm{kT}}\right]
$$

The relaxation strength $\Delta^{\mathrm{SK}}$ being identical to that of the original Schoeck model is consistent with various experimental facts about $\Delta^{\mathrm{SK}}$ that include: its magnitude, its temperature independence, and its increase with the amount of plastic deformation (see Figs. 5, 7, 8). In the present model the effect of interaction contributes to a reduced diffusivity $\mathrm{D}_{\text {Cottrell }}^{*}$ and to an enhanced damping constant $\mathrm{B}$, but it does not modify the response of the vibrating-string model used by Schoeck. The lower bound $\mathrm{H}^{\mathrm{S}}$ for $\mathrm{H}^{\mathrm{SK}}$ is favourable to make the model possibly consistent with the low value of $0.95 \mathrm{eV}$ found for the activation enthalpy in $\mathrm{Fe}-\mathrm{C} / 25 /$ and $\mathrm{n}=0.084$. 


\section{CONCLUSIONS}

The main experimental features of the SK relaxation in $\alpha$-Iron are demonstrated. The mechanical loss spectra in deformed $\alpha$-Iron can be more complicated then reported previously in the literature. For example, the $\mathrm{M}$ peak and splitting of the stable SK peak into two constituent $\mathbf{S K}_{1}$ and $\mathrm{SK}_{2}$ peaks are new observations. These peaks result from thermally unstable relaxation processes due to dislocation-FIAs interaction. The existence of the DESE in $\alpha$-Iron is proved. Two regimes of the diffusivity of FIAs in bcc metals are considered. The first is the Snoek relaxation, recognized as a primitive relaxation. The second is the coupled, cooperative diffusion of the FIAs in the Cottrell cloud which is characterized by a slowing of the relaxation rate relative to the primary Snoek relaxation. In other words, the Cottrell cloud is recognized to be a 'coupled cloud', that is, the relaxation is a many-body correlated interaction process. The relaxation parameters $\mathrm{H}^{\mathrm{SK}}, \tau^{\mathrm{SK}}$ and skewness of the SK peak are explained in terms of cooperative interaction of FIAs. In contrast to the Seeger and Schoeck models, the Magalas-Ngai model can explain the very low values of activation enthalpy of the SK peak observed in deformed $\alpha$-Iron doped with very small carbon concentration. Further theoretical progress can still be achieved. A new challenge in understanding of the SK relaxation is to include the stochastic character of the motion of dislocations and the cooperative migration of the FIAs in the Cottrell cloud.

\section{Acknowledgements}

This work is supported by the Polish State Committee for Scientific Research (KBN) under grant No. 7 T08B 04308 and by the Faculty of Metallurgy and Materials Science, University of Mining and Metallurgy under grant No. 10110370.

\section{References}

[1] Schoeck G., Acta Met., 11 (1963) 617 - 622; Schoeck G. and Mondino M., J. Phys. Soc. Japan, 18, Suppl.1, (1963) 149 - 152.

[2] Schoeck G., Scripta Metall., 10 (1982) 233 - 239.

[3] Schoeck G., Scripta Metall., 22 (1988) 389 - 394.

[4] Seeger A., Phys. Stat. Sol., (a) 55 (1979) 457 - 468.

[5] Seeger A., Journal de Physique, 42-C5 (1981) 201 - 228.

[6] Seeger A., Scripta Metall., 10 (1982) 241 - 247.

[7] Seeger A., Weller M., Diehl J., Pan Z.L. and Ke T.S., Z. Metallkde., 73 (1982) 1 - 20.

[8] Magalas L. B. and Ngai K. L., in Mechanics and Mechanisms of Material Damping, ASTM, STP 1304, 1996 (in press).

[9] Magalas L. B. and K. L. Ngai, to be published.

[10] Magalas L. B., Proc. Summer School on Internal Friction in Solids, June 1984, ed. Gorczyca S. and Magalas L. B., Wydawnictwo AGH, Kraków, Poland, 1984, pp. 89-130.

[11] Magalas L. B. and Gorczyca S., Journal de Physique, 46-C10 (1985) 253 - 256.

[12] Rubianes J., Magalas L. B., Fantozzi G. and San Juan J., Journal de Physique, 48-C8 (1987) 185-190

[13] Magalas, L. B. and Niblett D. H., Journal de Physique, 48-C8 (1987) $209-217$.

[14] Hirth T.P., Scripta Metall., 10 (1982) 221 - 223.

[15] Ke T.S., Scripta Metall., 10 (1982) 225 - 232.

[16] Ritchie I. G., Scripta Metall., 10 (1982) 249 - 253.

[17] Ngai K. L. and Magalas L. B., Materials Science Forum, 119-121 (1993) 49 - 60.

[18] Ngai K. L., Wang Y. N. and Magalas L. B., J. Alloys and Comp., 211/212 (1994) 327 - 332.

[19] Wang Y. N., Gu M., Sun L. and Ngai K. L., Phys. Rev. B, 50 (1994) 3525 - 3531.

[20] Nowick A. S. and Berry B. S., Anelastic Relaxation in Crystalline Solids, Academic Press, 1972.

[21] Magalas L. B., Introduction to Mechanical Spectroscopy, in Mechanical Spectroscopy, 1997, in press.

[22] Vanoni F., These, Universite de Grenoble, 1973.

[23] Magalas L. B., Measurement and Presentation of Mechanical Loss Spectra, in Mechanical Spectroscopy, 1997, in press.

[24] Magalas L. B., Dufresne J. F. and Moser P., Journal de Physique, 42-C5 (1981) 127 - 132.

[25] Iwasaki Y. and Hashiguchi K., Trans. Jap. Inst. Metals, 23 (1982) 243 - 249.

[26] Rubianes J., These, INSA de Lyon, France, 1990.

[27] Magalas L. B. and Fantozzi G., in Proc. of this Conference. 